\title{
Hiding digital image watermarking in a sound signal using Wavelet Transform and Singular Value Decomposition
}

\author{
Alyaa Moufaq Abdul Majeed Haleem \\ AlAA Zakaria \\ AlyaaHaleem@uomosul.edu.iq \\ College of Computers Sciences and Mathematics \\ University of Mosul, Mosul, Iraq
}

Received on: 04/09/2009

Accepted on: 16/05/2010

\section{ABSTRACT}

The outgrowing of multimedia applications in communications network brought about an increasing need to provide qualified methods to protect these media and secure communication. These methods include encryption and information hiding.

In this research, a new method has been improved to hide watermarking image in voice signal then send it through the network with no effect on the cover sound through the hiding process. This method is performed by applying two ways. The first one is the discrete wavelet transform (DWT) while the second one is singular value decomposition (SVD) by using wavelet transform, we get detail values which are arranged in a specific way and then entered to the singular value decomposition algorithm. Finally we get eign values, which embedding the data of the watermark image inside these values. So, we get the watermark signal with no effects. Also, the recovered image efficiency measurement is performed by using the correlation coefficient between the original and the recovered watermark image and also we apply the measurement of the difference between the original voice signal (cover) and the watermarked voiced signal by using signal to noise ratio (SNR). Matlab is the approval as a programming language in this paper.

Keywords: SVD, information Hiding, watermark, eign value.

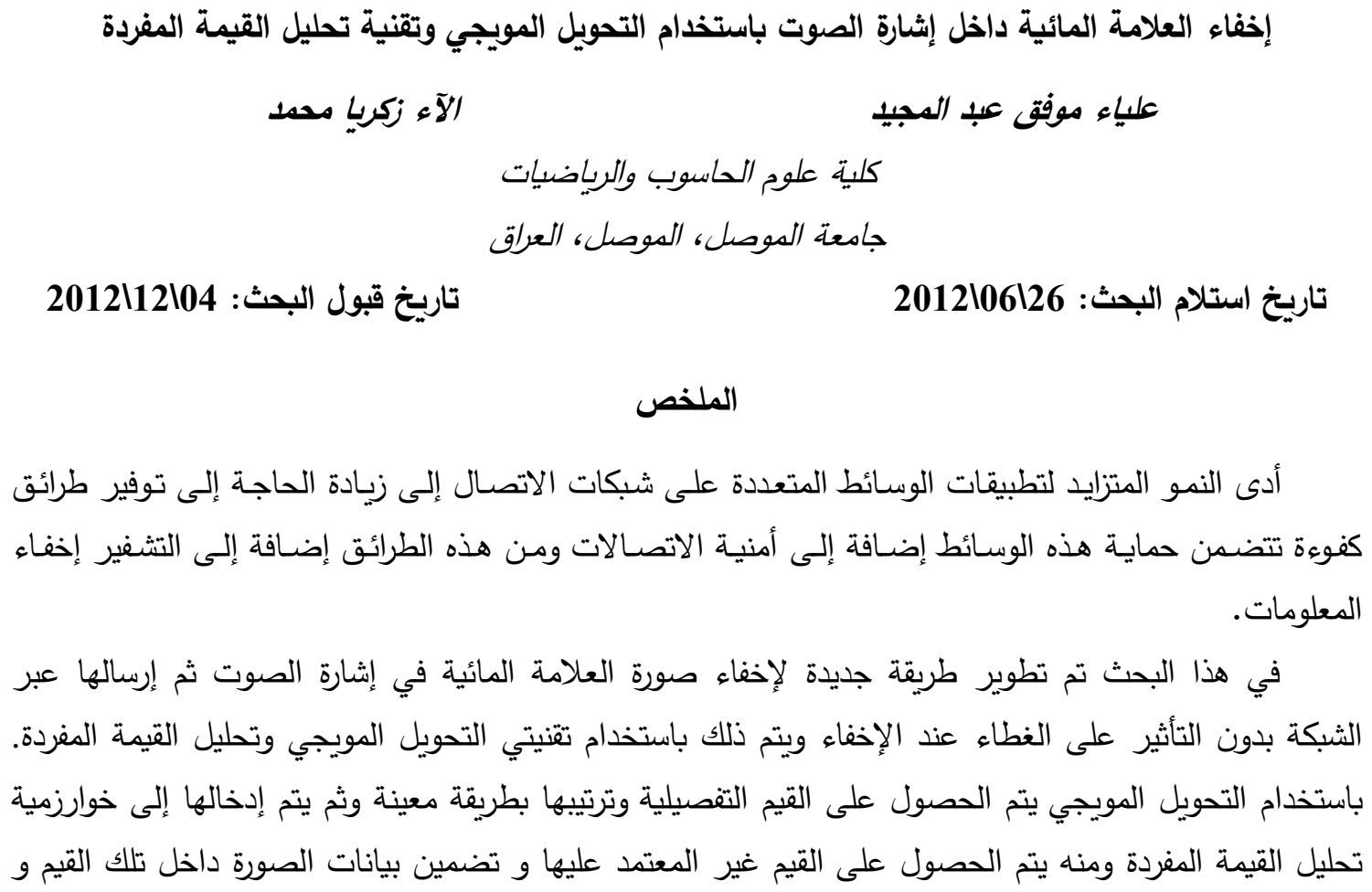


بهذه الطريقة يتم الحصول على الإثارة الصوتية بعد عملية التضمين بدون تأثير واضح عليها. كذلك تم قياس جودة الصورة المسترجعة باستخدام معامل الارتباط بين صورة العلامة المائية الأصلية والعلامة المائية المسترجعة وكذلك لكائك قياس مقدار الاختلاف بين إثارة الصوت الأصلي (الغطاء) والإثارة الصوتية بعد تضمين العلامة المائية باستخدام مقياس SNR. تم اعتماد Matlab كلغة برمجية في هذا البحث. الكلمات المفتاحية: تحليل القيمة المفردة ، اخفاء المعلومات ، العلامة المائية .

1. تمهيد

أدى النمو المتزايد لتطبيقات الوسائط المتعددة على شبكات الاتصال إلى زيادة الحاجة إلى توفير طرائق

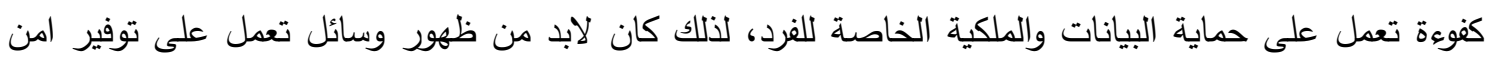
لهذه الوسائط لحمايتها من التحريف، السرقة أو نشر المعلومات الحساسة منها. ومن هنا ظهرت الحاجة إلى توفير وسائل أمنية البيانات، ومن هذه الوسائل علم التشفير Cryptography الذي يعنى بتوفير حماية لخزن البيانات

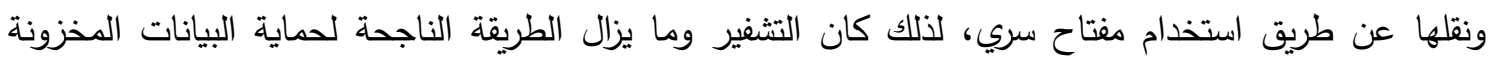

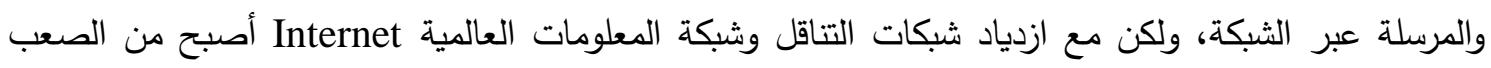
المحافظة على هذه البيانات، ولاسيما أنها دائما في متتاول الجميع عبر شبكة الانترنت في صيغة فيكة غير واضحة تبعث على الثك والاهتمام لدى المتطفل والسارق لفتح هذا التشفير أو تدمير المعلومات المرسلة.

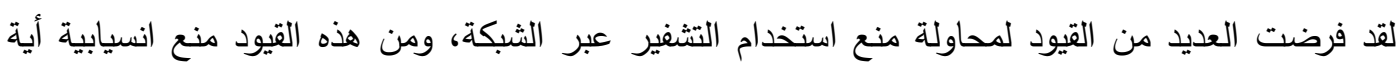

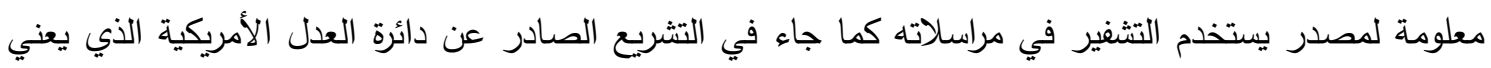
المقاطعة المعلوماتية، لذلك بدأت معظم الحكومات بفرض سيطرتها على التشفير ومنع المؤسسات من التعامل بهاه.

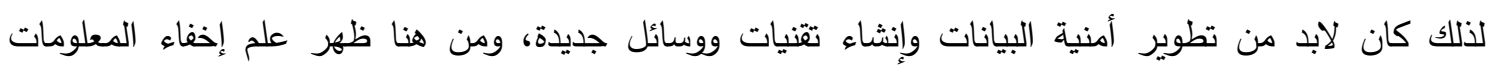

[2][1].information Hiding كان الإخفاء ومازال الطريقة الناجحة لحماية البيانات المخزونة والمرسلة عبر الثبكة لأنه يعطي أمنية عالية و يظهر عنصر الإخفاء دون أي تغيير • إن الغاية من إخفاء المعلومات ليست منع الآخرين من معرفة الإنة

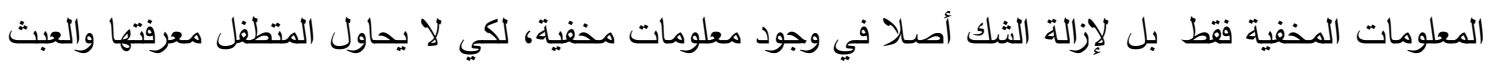
بها، والثيء المميز في تقنيات إخفاء المعلومات أنها تواكب التقنيات الحديثة، ويمكن استخدامها في جميع الوسائط

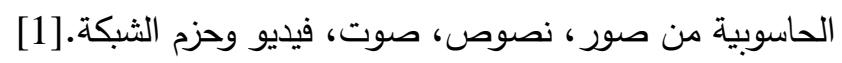
تعد العلامة المائية إحدى طرق الإخفاء المتعددة إذ زادت في الفترة الأخيرة ظاهرة التوزيع والنسخ غير

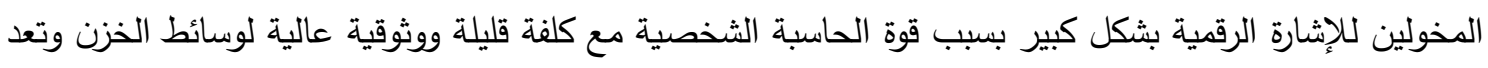
العلامة المائية إحدى وسائل حماية الحقوق الفكرية. 2. تعريف الإخفاء

يعرف إخفاء المعلومات على انـه تضمين بيانات سرية في أشكال مختلفة من الوسـائط المتعددة الرقميـة كملفات النصوص والصور والملفات الصوتية و الفيديويـة. وهذا يعني إخفاء (بيانات سرية) في معلومات أخرى لئي

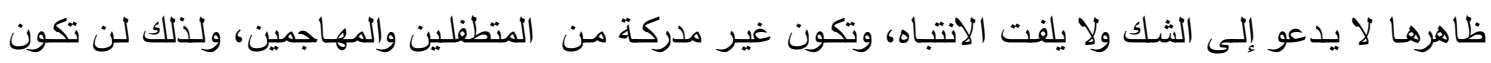

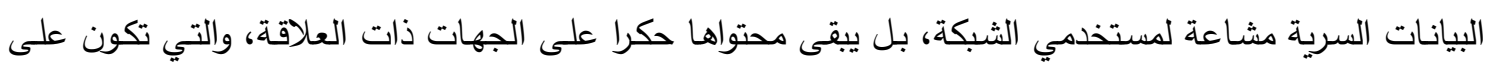


دراية بكيفية استخراج هذا المحتوى. ومع النمو السريع لتقنيات الشبكات والاتصالات، فان تقنيات الإخفاء أصبحت تستخدم بصورة واسعة لتحقيق أغراض متعددة منها حماية حقوق الطبع وتثبيت الملكية.[1]

3. خصائص متعارضة Conflicting Properties

$$
\text { ثمة خصائص يجب الأخذ بها عند تطبيق إحدى تقنيات إخفاء المعلومات، وهي: }
$$

• القدرة على عدم الإدراك (Imperceptibility): خاصية عدم تغيير الغطاء بصورة ملحوظة من قبل حواس وهئ الغطاء

$$
\text { الإنسان البصرية والسمعية. }
$$

• القوة (Robustness): وتعني مدى قوة تقنية إخفاء المعلومات أمام الهجمات عليها واكتثاف البيانات

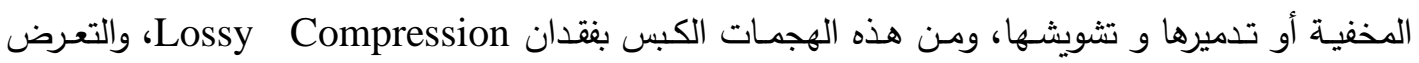

$$
\text { للمرشحات الخطية واللاخطية وغيرها من الهجمات. }
$$

• السعة (Capacity): كمية البيانات المراد إخفاؤها داخل وسط مضيف لونيف لها من دون كثفها. • القدرة على علم الاكتشاف (Undetected ability): وهذه الخاصية تعني إن البيانات المخفية تكون غير قابلة للكثف من المهاجمين.

إن الخصائص السابقة تكون متعارضة فيما بينها ولا يمكن تحقيقها جميعا في وقت واحد، ومثال ذلك إن نخفي معلومات كبيرة داخل صورة، فعند ذلك لا نحقق القوة المطلوبة ويكون من السهل اكتثاف إن ثمة بيانات

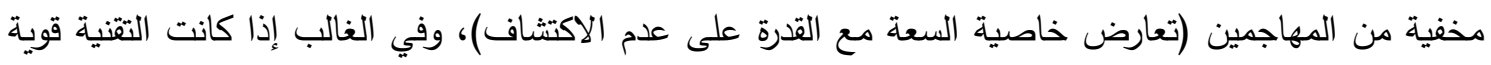
ضد الهجمات والتشويش عليها فان كمية البيانات المخفية ستكون قليلة، وغالبا ما يجب توفير خاصية القوة في تقنيات إثبات الملكية لان تلك التقنيات تتطلب القوة ضد الهجمات ويقابل ذلك إخفاء بيانات قليلة نسبيا (تعارض

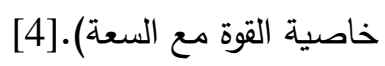

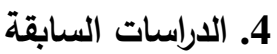

حاول الباحثون إيجاد تقنيات إخفاء متطورة تواكب التطور السريع في تقنيات الإخفاء والثبكات، فمنهم دن أنجز بحوثا ودراسات واسعة لربط تقنيات إخفاء المعلومات مع تقنيات الذكاء الاصطناعي، وآخرون أنجزوا الاتصال السري في شبكات الحاسوب من خلال القنوات المخفية، ومن هذه البحوث:

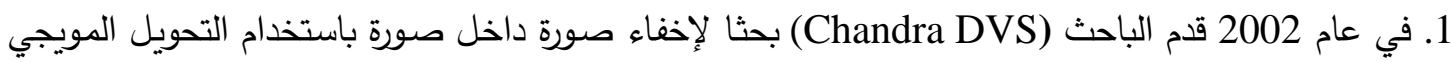
وتحليل القيمة المفردة عن طريق دمج SVs للصورة الأصلية مع SVs لصورة العلامة المائية باستخدام عامل

$$
\text { تحجيم ثابت. }
$$

2. في عام 2009 قدم الباحثون Amansouri,A mahmoudi Aznaveh and Ftorkamani) بحثابك (CWT) Complex Wavelet لإخفاء صورة العلامة المائية داخل ملف غطاء من نوع صورة باستخدام transform و تقنية تحليل القيمة المفردة.[5] 3. في عام 2010 قدم الباحثان (Al_Haj Ali and Mohammad Ahmad) بحثا لإخفاء صورة العلامة المائية الثنائية داخل ملف صوتي عن طريق تطبيق أربعة مستويات للتحويل المويجي على كل مقطع من مقاطع الإشارة الصوتية وإعادة ترتيب المعاملات التفصيلية داخل مصفوفة جديدة ثم تطبيق خوارزمية تحليل 
القيمة المفردة على المصفوفة الناتجـة لينتج ثـلاث مصفوفات هي U,S,V يتتم تضـمين قيم البـت للعلامـة المائية W(n) داخل العنصر الأول لقطر المصفوفة S حسب المعادلة الآتية: $S W_{11}=S_{11} \times\left(1+\left(\alpha \times W_{(n)}\right)\right)$

إذ أن: W(n) : تمثل مصفوفة بيانات العلامة المائية بالنظام الثنائي.

S تمثل العنصر الأول للقطر الرئيسي داخل المصفوفة S:

ب بعد تضمين قيمة بت من العلامة

المائية، أما قيمة $0.2=0$.

حيث تم إخفاء صورة ثنائية (Binary image) بحجم 6*4 ـ ـ أما في عملية الاسترجاع فيجب وجود ملف الغطاء الأصلي بالإضافة إلى الملف المضمن إذ يتم إيجاد قيمة البت للعلامة المائية المضمنة داخل كل مقطع

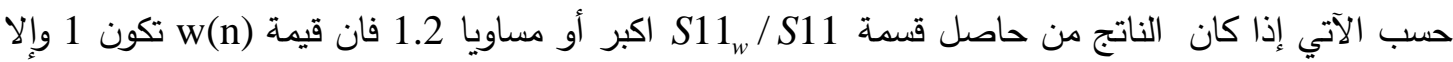
[3] [3(n) مساوية للصفر

\section{Wavelet Transform التحويل المويجي 5}

إن القصور في تمثيل الإشارة باستخدام تحويلات فورير كان السبب وراء ظهور تقنيات التحويل المويجي، إذ أن تحويل فورير يظهر الترددات التي حدثت في الإشارة دون الإشارة إلى زمن حدوثها وهذا التشثيل لا يكون كافيا

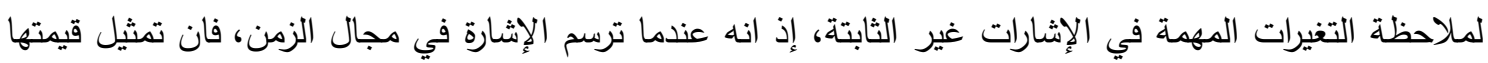

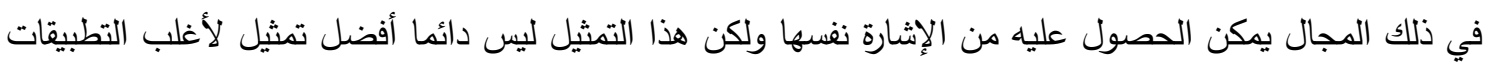

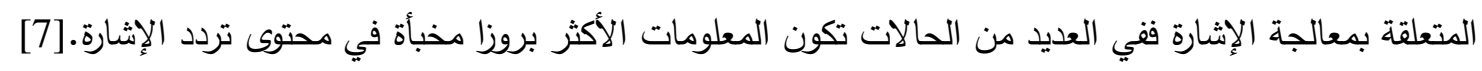

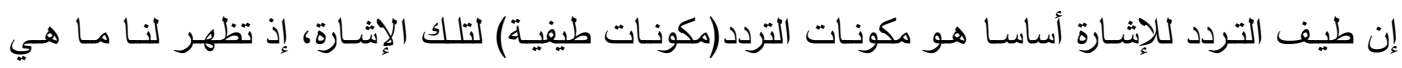

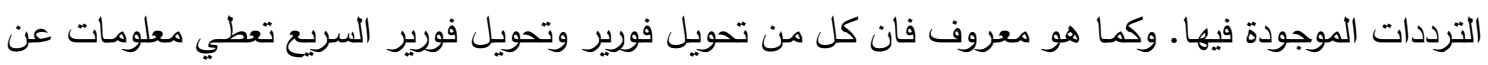

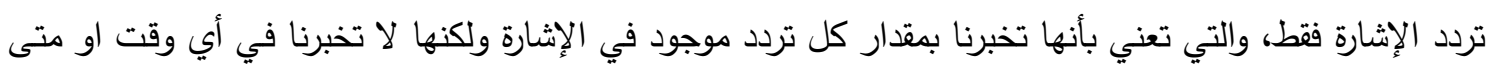

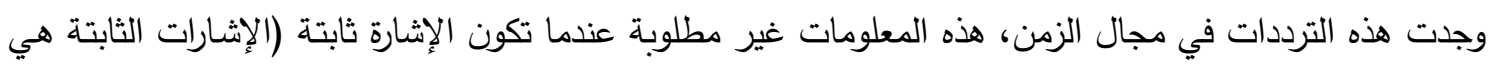

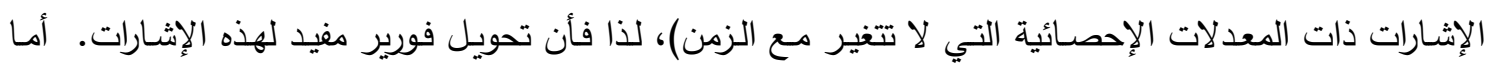

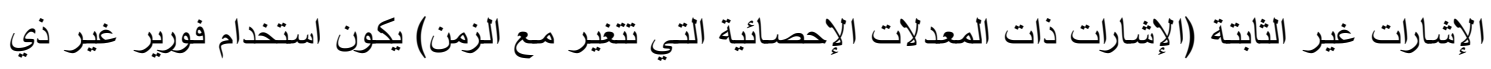

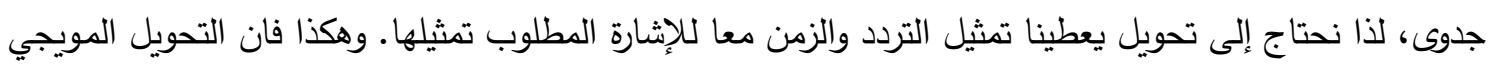

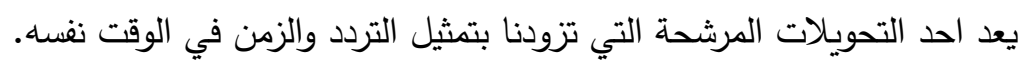

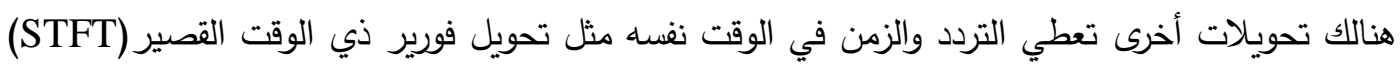

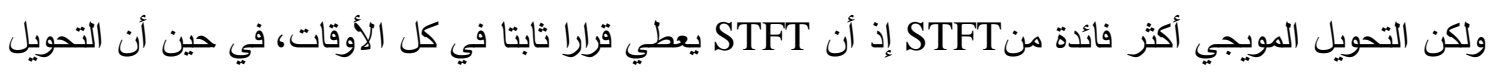

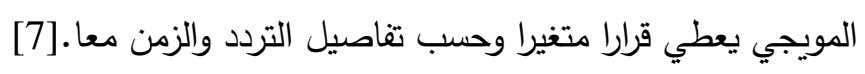

كما ويتميز التحويل المويجي على تحويلات فورير بميزات أخرى، فعلى سبيل المثال يعمل التحويل المويجي

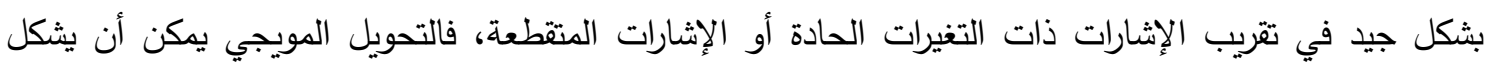

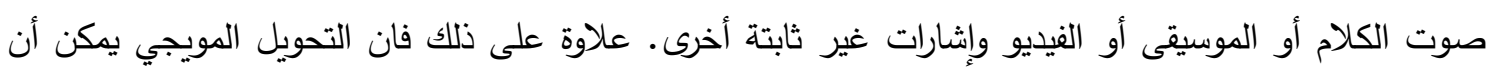
يستعمل في العديد من التطبيقات مثل إزالة الضوضاء من الصور أو كبس الصور أو تحسين الرؤية لمشهد معين يخص الإنسان.[3] 
تتم عملية التحليل عن طريق إمرار الإشارة (Signal) على مرشحين الأول هو مرشح الإمرار الواطئ

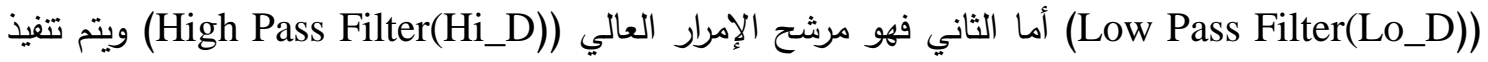
عملية اللافوف الرياضي (Convolution) وعملية التنقيص الثنائي Down sampling على إخراج المرشحين بذلك يتم الحصول على معاملات التقريب Approximation coefficients cA(n) من مشرح الإمرار الواطئ (Lo_D) والحصول على معاملات التفصيل (Hi_D من مرشح الإمرار العالي( Detail coefficients cD(n)

كما موضح في الثكل(1).

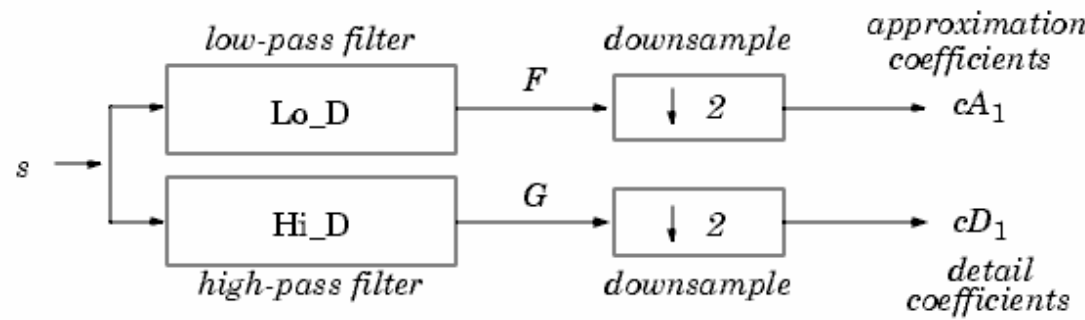

where:

$$
\mathrm{X}
$$

Convolve with filter $\mathrm{X}$.

2 Keep the even indexed elements

الثكل (1) يوضح مرحلة التحليل للتحويل المويجي المقطع

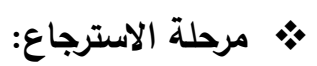

يتم استرجاع معاملات التقريب (n) بواسطة الاستكمال أي عن طريق حشر أصفار بين كل عينتين من عينات التقريب cA $\mathrm{CA}_{\mathrm{j}}(n)$ وعينات التفصيل أيضا ومن ثم إدخالها إلى مرشحات الاسترجاع (Hi_R) (Lo_R)

في الثكل (2). (2)

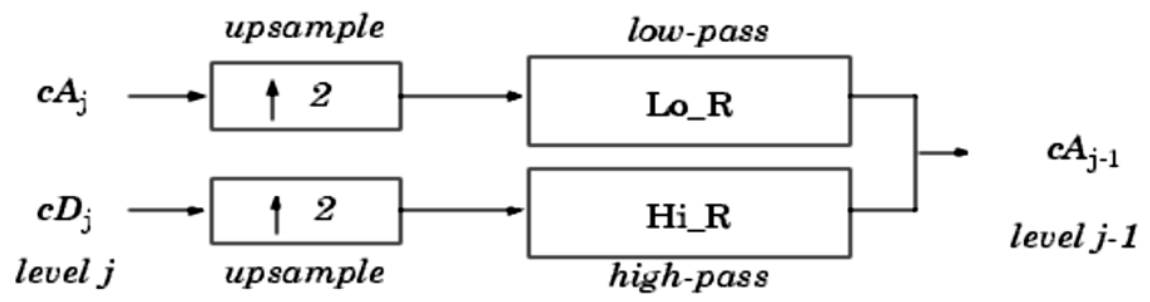

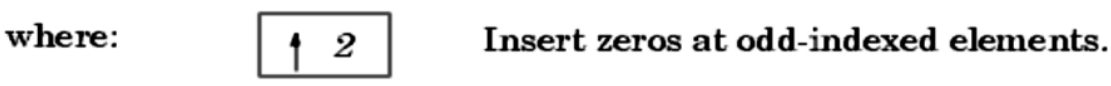

$\mathrm{X} \quad$ Convolve with filter $\mathrm{X}$.

الثكل (2) يوضح مرحلة الاسترجاع للتحويل المويجي المقطع

حيث نلاحظ أن التحويل المويجي المقطع لا يحتوي على عمليات تكامل أو عمليات اشتقاق وإنما تحتوي

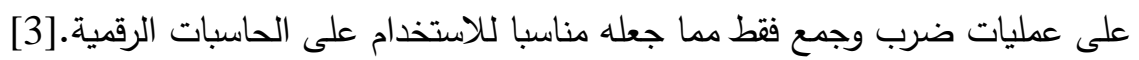




\section{6. تحليل القيمة المفردة Single Value Decomposition (SVD)}

إن تقنية (SVD) مبنية على نظريات الجبر الخطي والتي تتضمن تقسيم المصفوفة المستطيلة A إلى ثلاث مصفوفات، المصفوفة المتعامدة (U) وهي المصفوفة التي يكون حاصل ضربها في المدور لها هو مصفوفة قطرية جميع عناصر القطر لها 1 ، المصفوفة القطرية (S) هي المصفوفة التي تكون جميع عناصرها أصفارا ماعدا عناصر القطر والمصفوفة المتعامدة (V) هي المصفوفة التي يكون حاصل ضربها في المدور لها هو مصفوفة قطرية جميع عناصر القطر لها 1. $\mathrm{A}_{\mathrm{mn}}=\mathrm{U}_{\mathrm{mm}} \mathrm{S}_{\mathrm{mn}} \mathrm{V}^{\mathrm{T}}{ }_{\mathrm{nn}}$

حيث أن الأعمدة للمصفوفة u والتي تمثل القيم الذاتية للمصفوفة A A هي Orthonormal ( يطلق على المتجـه الذي يكون طولـه وحدة طـول واحـدة وحاصـل ضـربه مـع كل vector ضـــ المصنفوفة يسـاوي صـفراً Orthonormal هي v v وتسمى المصفوفة في ذلك الحين مصفوفة متعامدة والأعمدة للمصفوفة (Orthonormal

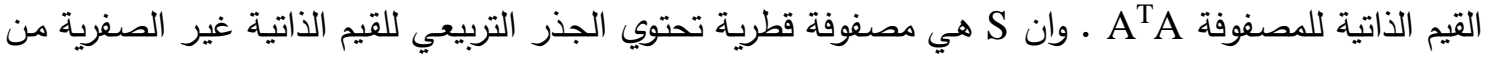

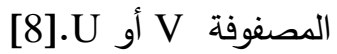

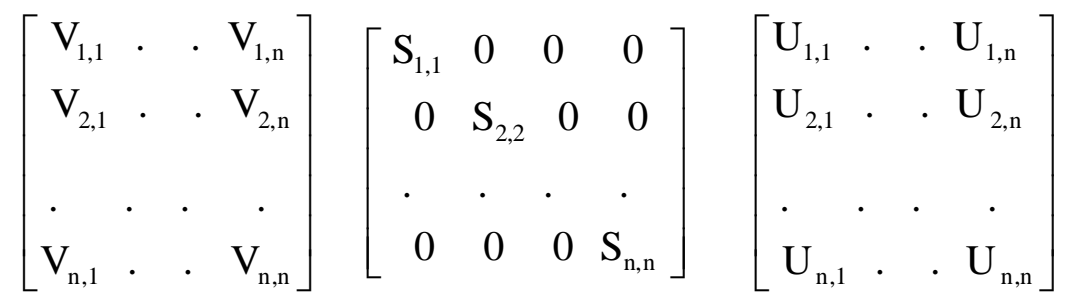

7.

في هذه الطريقة يتم استخدام التحويل المويجي وتحليل القيمة المفردة إذ يتم الإخفاء باستخدام المصفوفة

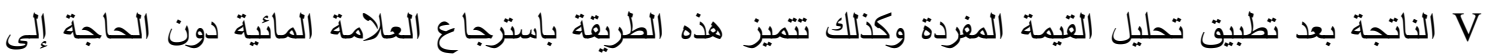
ملف الغطاء الأصلي. وتتلخص هذه الخوارزمية بالخطوات الآتية: 1.7 خوارزمية الإخفاء المدخلات: ملف الغطاء (ملف صوتي)، صورة العلامة المائية المراد إخفاؤها (أي صورة ملونة أو رمادية من نوع . أوBPG BMP المخرجات: الملف الصوتي بعد إخفاء المعلومات. الخطوة الأولى: إدخال اسم ملف الغطاء، ثم قراءة محتويات الملف وخزن العينات الصوتية في مصفوفة X.

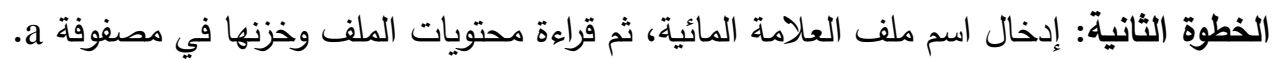

الخطوة الثالثة: تقسيم الإثارة الصوتية x إلى مجموعة من القوالب (Frame) حيث كل قالب بحجم 512 (الثينة. الخطوة الرابعة: إجراء عملية تغير لأبعاد الصورة a بحيث تلائم حجم الملف الصوتي الذي تم اختياره لإخفاء الصورة بالاعتماد على الصيغة التالية الطول/العرض للصورة = 
الخطوة الخامسة: تحويل مصفوفة الصورة للعلامة المائية a إلى مصفوفة ذات بعد واحد ومن ثم تحويل القيم اللونية إلى النظام الثنائي ومن ثم تحويل المصفوفة الأخيرة إلى مصفوفة ذات بعد واحد أطلق عليها مصفوفة

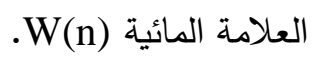
الخطوة السادة: تتفيذ أربعة مستويات للتحويل المويجي على كل قالب لينتج لدينا ويمثلون مصفوفات المعاملات التصيلية لكل مستوى وكذلك ينتج cA وهي مصفوفة المعاملات التقريبية[3]، كما موضح في الثكل(3).

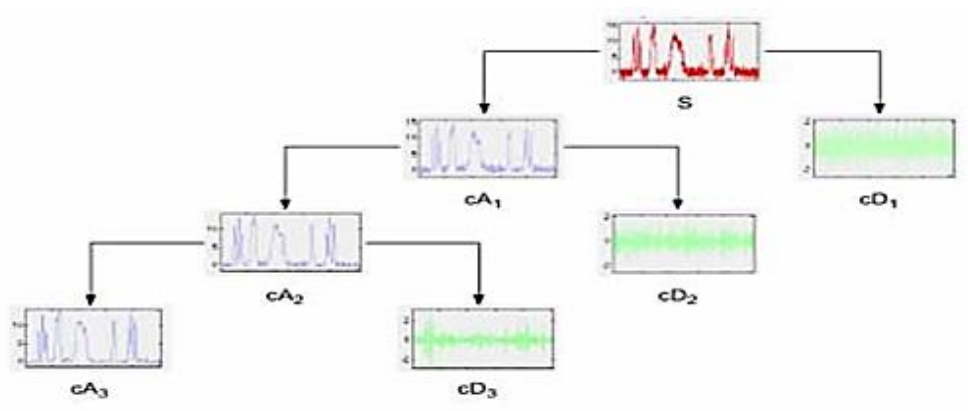

الثكل (3) يوضح نتائج التحليل المويجي لقالب من الإثارة لثلاثة مستويات

الخطوة السابعة: ترتيب مصفوفات المعلومات التقصلية الناتجة من الخطوة السابقة بالطريقة التالية حيث أطلق

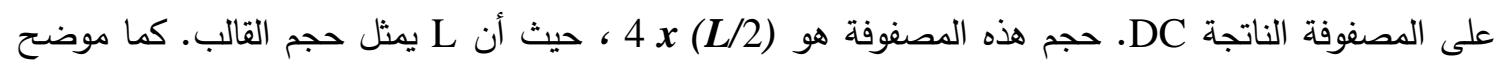
في الثكل (4)

\begin{tabular}{|l|c|c|c|c|c|c|c|}
\hline \multicolumn{2}{|c|}{$c D_{1}$} \\
\hline \multicolumn{2}{|c|}{$c D_{2}$} & \multicolumn{3}{c|}{$c D_{2}$} \\
\hline \multicolumn{2}{|c|}{$c D_{3}$} & \multicolumn{2}{|c|}{$c D_{3}$} & \multicolumn{2}{c|}{$c D_{3}$} & \multicolumn{2}{c|}{$c D_{3}$} \\
\hline$c D_{4}$ & $c D_{4}$ & $c D_{4}$ & $c D_{4}$ & $c D_{4}$ & $c D_{4}$ & $c D_{4}$ & $c D_{4}$ \\
\hline
\end{tabular}

الثكل (4) يوضح ترتيب مصفوفة DC

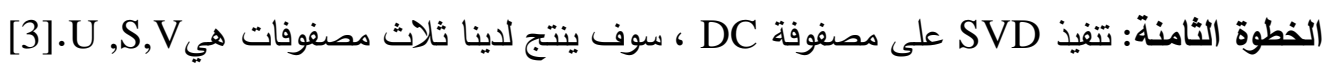

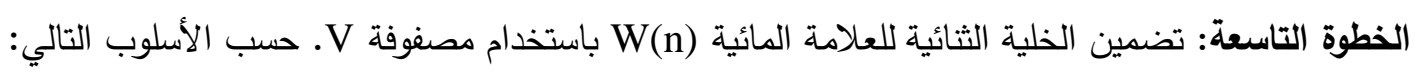
y=1/V(i,j) لتضمين قيمة بت واحد بداخله حيث يتم إيجاد معكوس القيمة ل • تضمين بت من العلامة المائية داخل الجزء الصحيح للعدد y الناتج من القسمة بعد تحويله إلى الصيغة لدئة الثنائية إذ يتم استبدال $W_{i}$ مع قيمة البت الثامن للعدد y (إخفاء داخل الخلية الثنائية الأعلى أهمية.

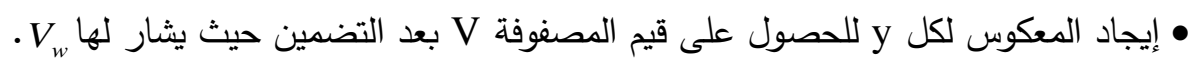

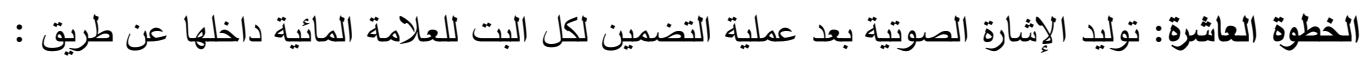
تطبيق معكوس تحليل القيمة المفردة للحصول على مصفوفة DC الناتجة من عملية التضمين. $D C_{w}=U \times S \times V_{w}^{T}$

تتفيذ أربع مستويات من التحويل المويجي المعكوس على مصفوفة DC للحصول على القالب بعد التضمين وأخيرا تجميع القوالب للحصول على العينات الصوتية بعد تضمين العلامة المائية 


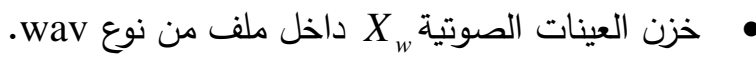

و الثكل (5،أ وب) يوضح المخطط الانسيابي لأسلوب الإخفاء للطريقة المطورة. أما الثكل(6) فئسات فيوضح المخطط الانسيابي لدالة التضمين المستخدمة في الطريقة المطورة.

- أ

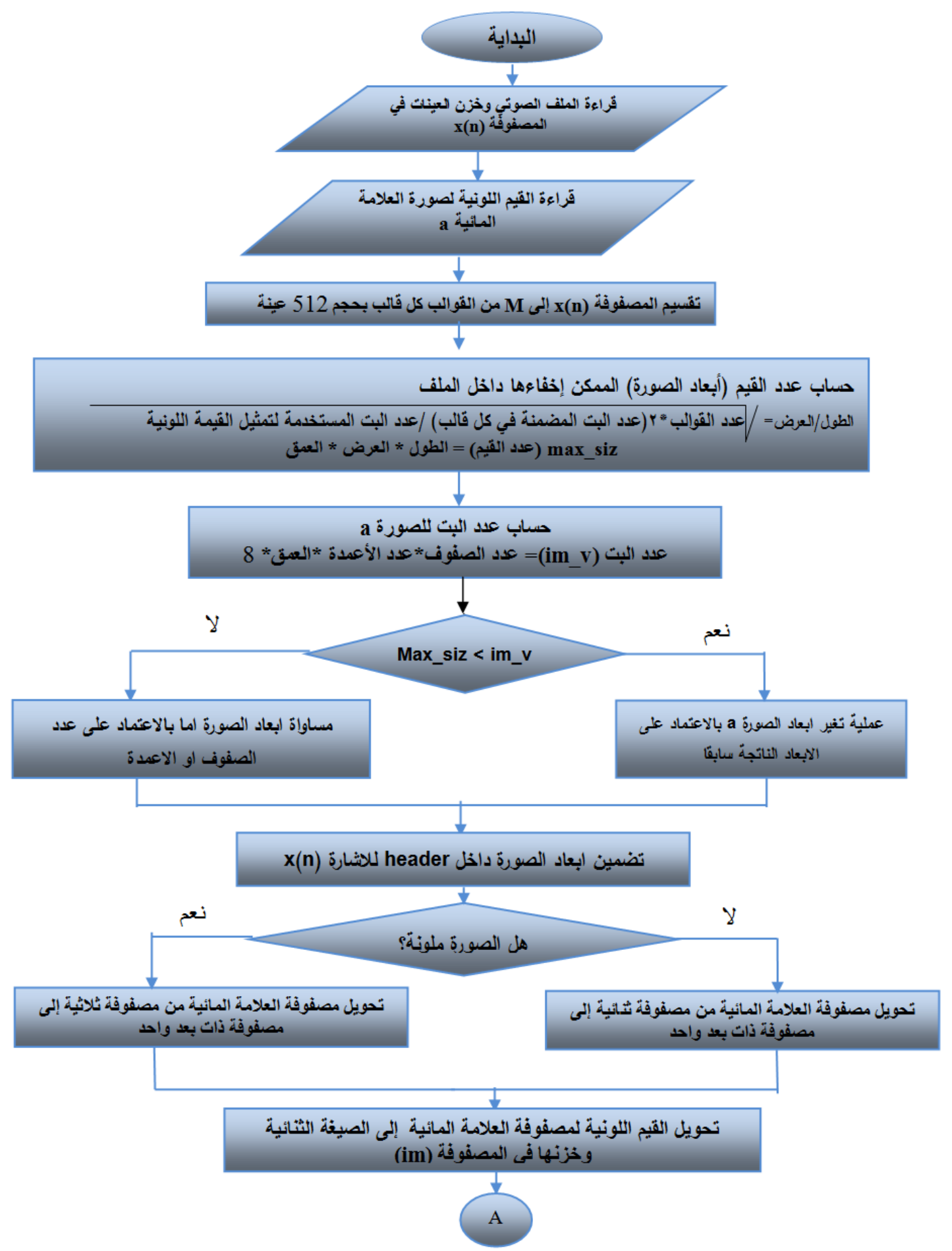

الثكل (5،أ و ب) مخطط انسيابي يوضح أسلوب الإخفاء للطريقة المطورة 


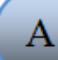

A

no bit حساب عدد البت لمصفوفة العلامة المائية

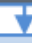

$\mathbf{i}=\mathbf{1}$

$$
4
$$

أتطبيق اريع مستويات من التحويل المويجي المقطع للقالب

$\checkmark$

توليد مصفوفة DC(m,n) من مصفوفات المعاملات التثصيلية

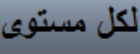

$\checkmark$

استدعاء دالة تحليل القيمة المفردة على مصفوفة DC لينتج $\mathrm{U}(\mathrm{m}, \mathrm{m}), \mathrm{S}(\mathrm{m}, \mathrm{n}), \mathrm{V}(\mathbf{n}, \mathbf{n})$

V ايجاد مدور المصفوفة $\mathrm{V}=\mathrm{V}^{\mathrm{T}}$

$\checkmark$

استدعاء دالة التضمين داخل المصفوفة V للمحتويات الموقع

استاعاء دالة التضمين داخل المصفوفة Vللمحتويات الموقع (1,32 $\vdash$

U,S,V تطبيق معكوس تحليل القيمة المفردة على المصفوفة

تطبيق معكوس التحويل المويجي

$\checkmark$

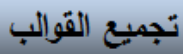

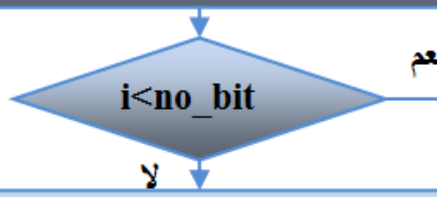

تحويل مصفوفة القوالب الثثائبة البعد إلى مصفوفة ذات بعد واحد

خزن الاشارة الناتجة بعد التضمين

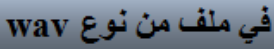

النهاية 


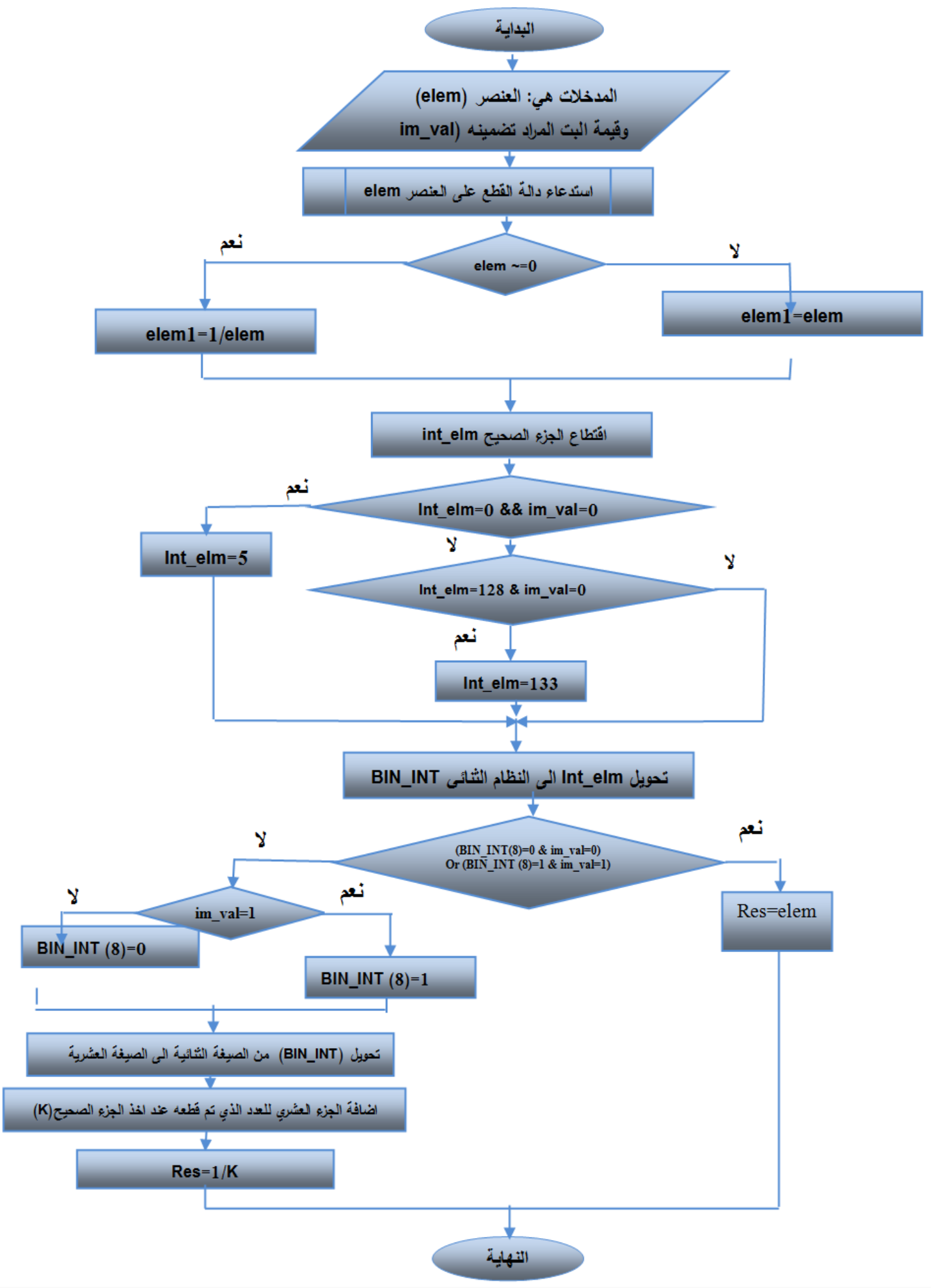

الثكل (6) مخطط انسيابي يوضح دالة التضمين للخوارمية المطورة 
2.7 خوارزمية الاسترجاع

المدخلات: الملف الحاوي على العلامة المائية المضمنة .

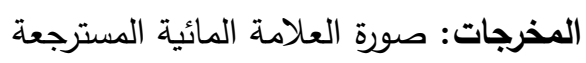
الخطوة الأولى: إدخال اسم الملف الحاوي على العلامة المائية المضمنة، ثم قراءة محتويات الملف وخزن العينات

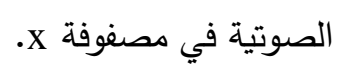

الخطوة الثانية: تقسيم الإشارة الصوتية x إلى مجموعة من القوالب (Frame) حيث كل قالب بحجم 512 عينة.

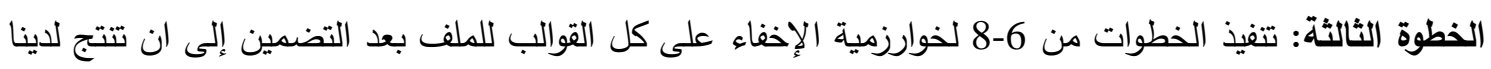
Uw, الخطوة الرابعة: استرجاع القيم الثنائية للعلامة المائية W(n) باستخدام مصفوفة V .حسب الأسلوب التالي:

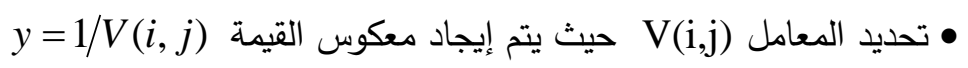

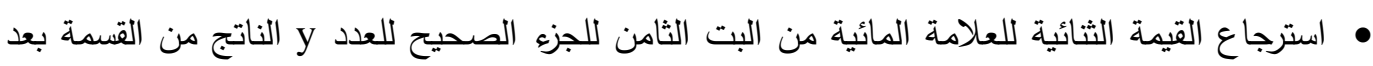

تحويله إلى الصيغة الثنائية.

الخطوة الخامسة: تحويل قيم الصورة من الثنائي إلى الصيغة العشرية. وتكوين الصورة وعرضها.

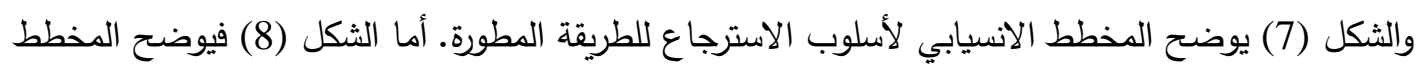
الانسيابي لدالة الاسترجاع المستخدمة في الطريقة المطورة. 


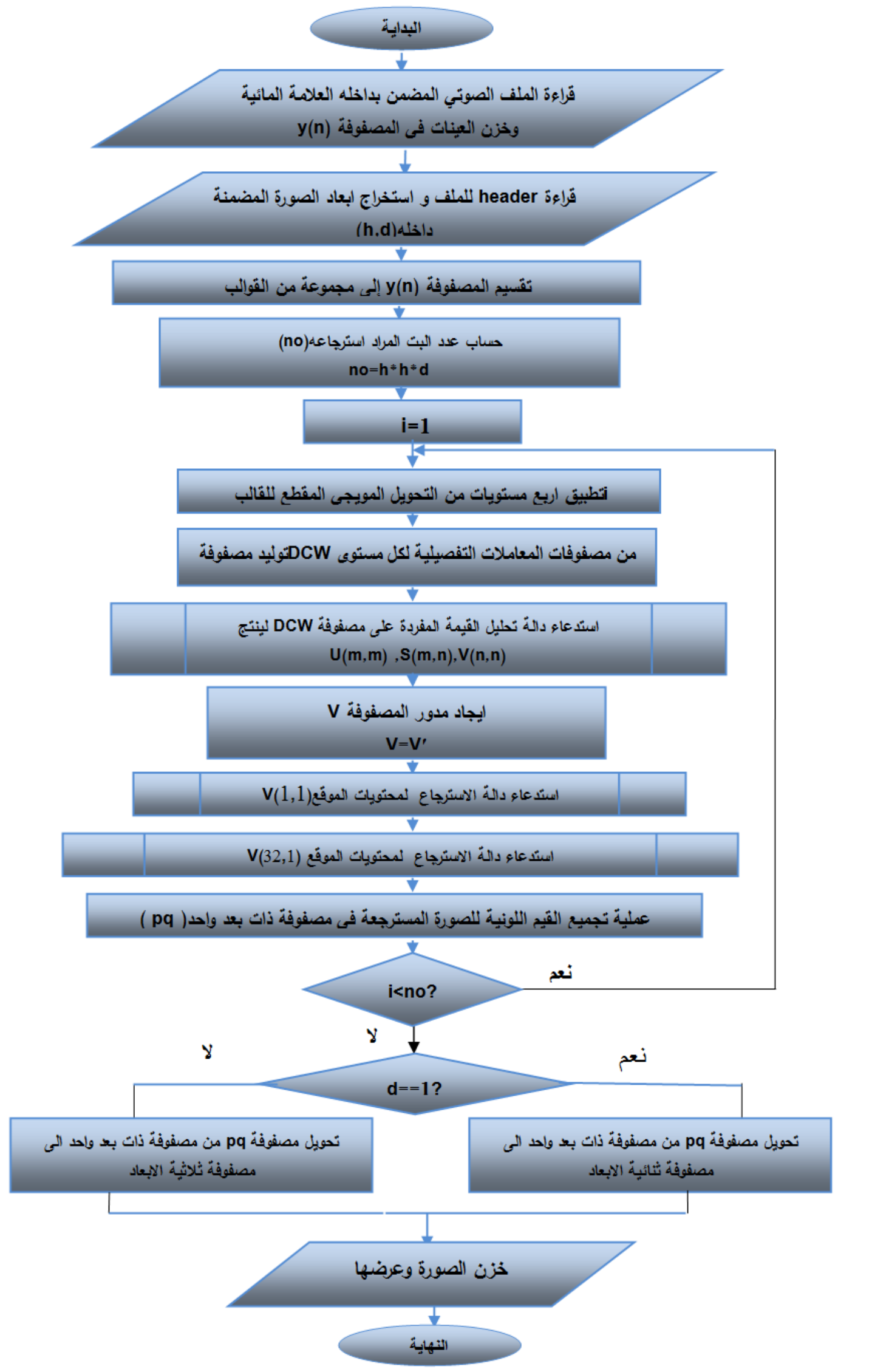

الثكل (7) مخطط انسيابي يوضح أسلوب الاسترجاع للطريقة المطورة 


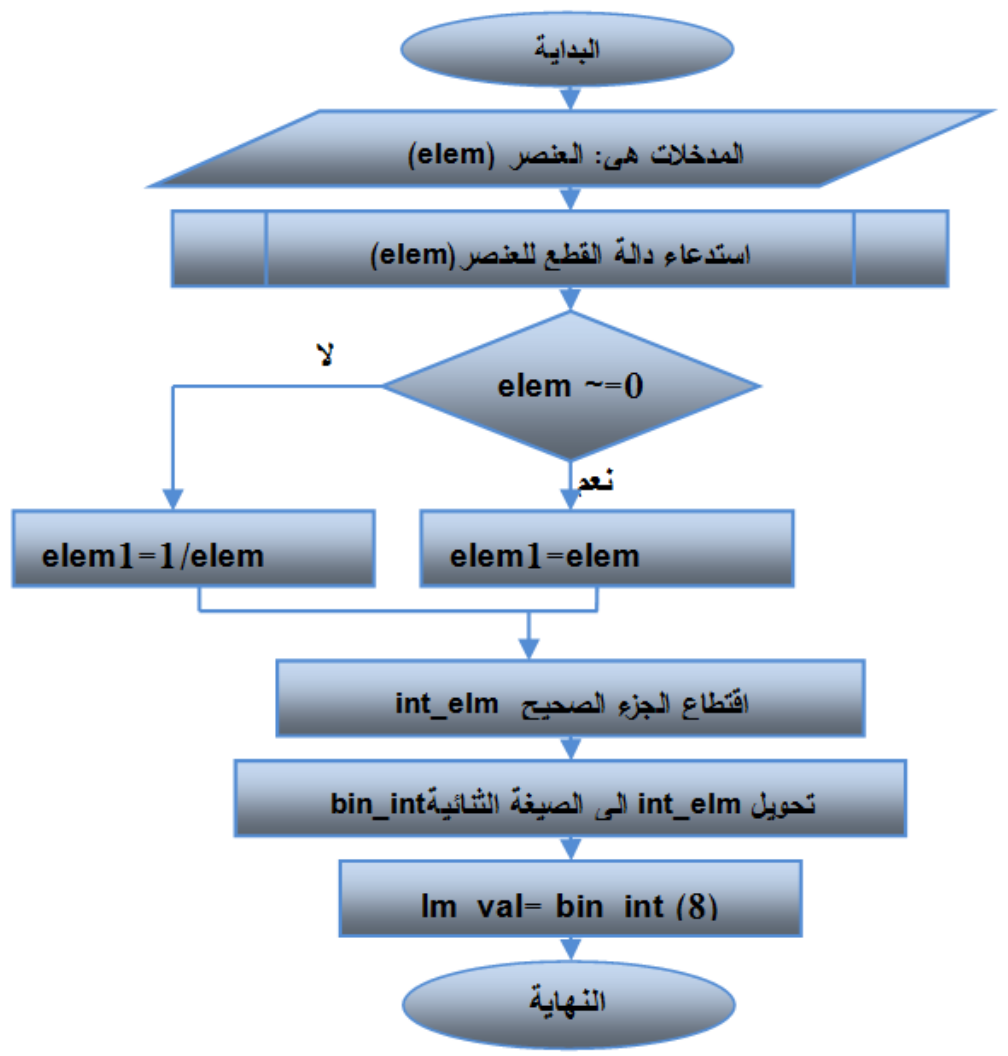

الثكل (8) مخطط انسيابي يوضح دالة الاسترجاع للطريقة المطورة

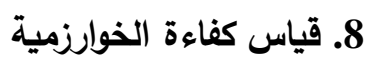
1.8 قياس مقدار الاختلاف

يتم قياس كفاءة الخوارزمية من خلال تقييم ومقارنة جودة الكلام بعد إجراء المعالجة المطلوبة بواسطة بعض Subjective and Objective ) أنواع المقاييس، التي استخدمت نوعين من الاختبارات الشخصية والموضوعية Test

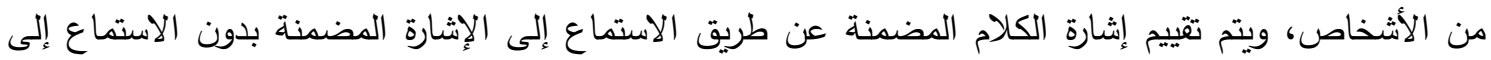

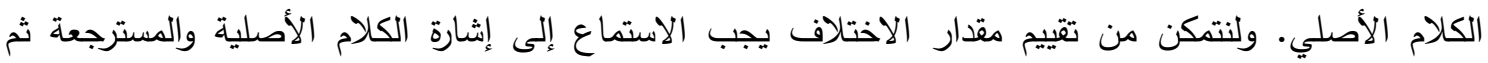

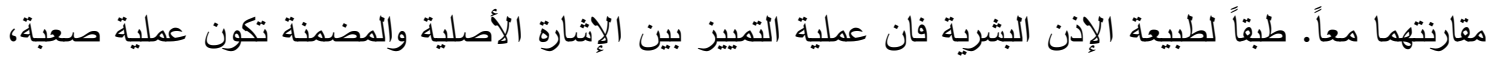

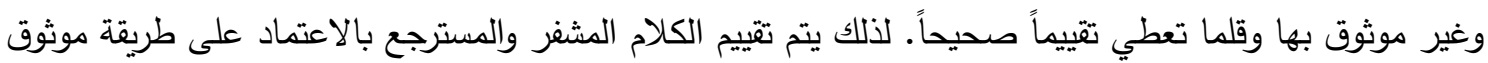
بها وهي الاختبارات الموضوعية (Test of Speech Signal Objective). لمعرفة مقدار التغيير الحاصل بين الإثارة الصوتية الناتجة بعد تضمين العلامة المائية والإشارة الصوتية الأصلية (إثارة الغطاء) يتم الاعتماد على المقاييس الموضوعية ومنها مقياس SNR (signal to noise ratio)، حيث تعرف بأنها نسبة الاختلاف بين الإثارة الحاوية على ضوضاء والإشارة الأصلية. تسمى وحدة قياس SNR بالديسبل dB وتكون النسبة بين هي نسبة جيدة وتعبر هذه القيمة عن خاصية قدرة عدم الاكتثاف Undetected ability)

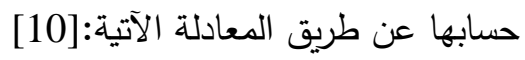


$S N R=\frac{1}{M} \sum_{m=1}^{M} 10 \log _{10} \frac{\sum_{n=1}^{N} \mid \text { org_sig }\left.(n)\right|^{2}}{\sum_{n=1}^{N} \mid \text { wat_sig(n)-org_sig }\left.(n)\right|^{2}}$

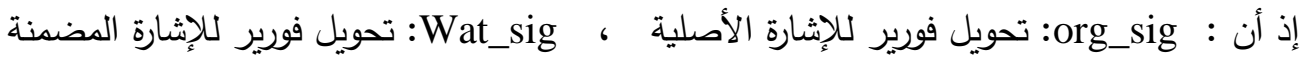
N

2.8 قياس جودة العلامة المائية المسترجعة

يتم حساب نسبة التطابق بين صورة العلامة المائية الأصلية والمسترجعة بالاعتماد على معامل الارتباط correlation factor اثثين من المتغيرات (X) و (Y) معرف كما يأتي: $\rho(X, Y)=$ Covariance $(X, Y) /\{\text { Variance }(X) \times \text { Variance }(Y)\}^{1 / 2}$

حيث إن (م) هو معاملُ الارتباط، وهو العدد الذي يُلخّصُ الاتجاه وقربَ العلاقاتِ الخطيّة بين متغيّرين. ومعامل

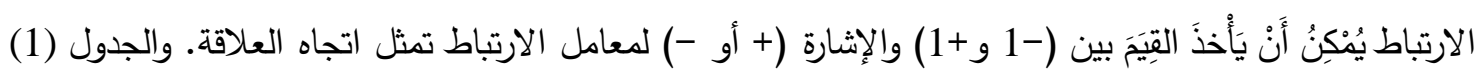
يصف القوة والاتجاه لقيم معامل الارتباط. الجدول (1) قوة واتجاه قيم معامل الارتباط

\begin{tabular}{|c|c|c|}
\hline الاتجاه & 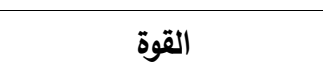 & معامل الارتباط(P) \\
\hline+ & علاقة خطية مثالية & $\mathbf{P}=1$ \\
\hline+ & علاقة خطية قوية جداً & $1>\mathbf{P}>=0.9$ \\
\hline+ & علاقة خطية قوية & $0.9>\mathbf{P}>=0.7$ \\
\hline+ & علاقة خطية متوسطة & $0.7>\mathbf{P}>=0.5$ \\
\hline+ & علاقة خطية ضعيفة & $0.5>\mathbf{P}>=0.3$ \\
\hline \multirow[t]{2}{*}{+} & علاقة خطية ضعيفة جداً & $0.3>\mathbf{P}>0$ \\
\hline & لا توجد علاقة & $\mathbf{P}=0$ \\
\hline- & علاقة خطية ضعيفة جذاً & $0>\mathbf{P}>-0.3$ \\
\hline- & علاقة خطية ضعيفة & $-0.3>=\mathbf{P}>-0.5$ \\
\hline- & علاقة خطية متوسطة & $-0.5>=\mathbf{P}>-0.7$ \\
\hline- & علاقة خطية قوية & $-0.7>=\mathbf{P}>-0.9$ \\
\hline- & علاقة خطية قوية جداً & $-0.9>=\mathbf{P}>-1$ \\
\hline- & علاقة خطية مثالية & $\mathbf{P}=-1$ \\
\hline
\end{tabular}

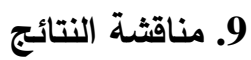

أجريت اختبارات متعددة على الخوارزمية المطورة لتضمين صورة مختلفة الأبعاد رمادية وملونة

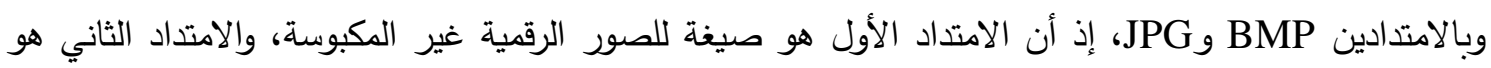

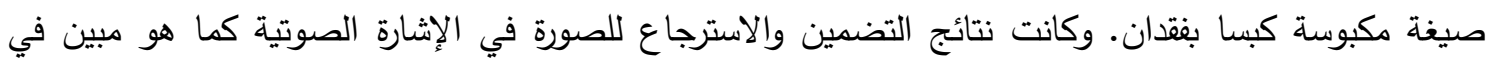

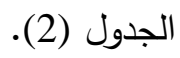


الجدول (2) نتائج التضمين للخوارزمية المطورة

\begin{tabular}{|c|c|c|c|c|c|}
\hline \multicolumn{2}{|c|}{ قياس كفاءة الخوارمية } & \multirow[b]{2}{*}{ العلامة } & \multirow[b]{2}{*}{ حجم الغطاء } & \multirow[b]{2}{*}{ صورة العلامة المائية } & \multirow[b]{2}{*}{ الصوتي(الغطاء) } \\
\hline $\begin{array}{c}\text { CORR } \\
\text { الصورة الأصلية/الصورة } \\
\text { المسترجعة }\end{array}$ & $\begin{array}{c}\text { SNR } \\
\text { الأصلية/الإشارة } \\
\text { المضمنة }\end{array}$ & & & & \\
\hline 1 & 41.0049 & $64 * 64 * 1$ & 8. $06 \mathrm{MB}$ & L_map.bmp & $\begin{array}{c}\text { Loopmusic1.wa } \\
\mathbf{v}\end{array}$ \\
\hline 1 & 30.41 & $64 * 64 * 1$ & $8.06 \mathrm{MB}$ & $\begin{array}{c}\text { Cameraman.b } \\
\text { mp }\end{array}$ & $\begin{array}{c}\text { Loopmusic1.wa } \\
\mathbf{v}\end{array}$ \\
\hline 0.87431 & 28.2127 & $21 * 21 * 3$ & $5.509 \mathrm{~KB}$ & Children.bmp & Loopvlong.wav \\
\hline 0.95258 & 29. 4931 & $30 * 30 * 3$ & $8.06 \mathrm{~KB}$ & Swar117.jpg & $\begin{array}{c}\text { Loopmusic1.wa } \\
\mathbf{v}\end{array}$ \\
\hline 0.9756 & 29.6421 & $21 * 21 * 3$ & $5.509 \mathrm{~KB}$ & Smallimage.jpg & Loopvlong.wav \\
\hline
\end{tabular}

ويبين الجدول (2) قياس مقدار الاختلاف بين الإشارة الأصلية والإشارة بعد التضمين حسب المعادلة (2)

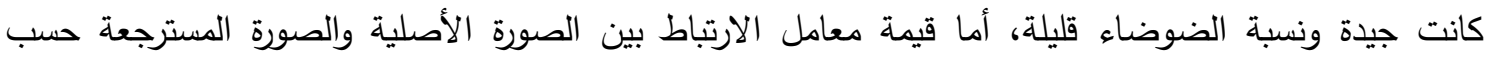
المعادلة (3) فان النتيجة كانت جيدة وهي علاقة خطية قوية جداً حسب الجدول (1). يوضح الثكل (9)، (10) (10) و (11) أنموذجا لصورة رمادية وملونة من الصور التي تم التطبيق عليها.

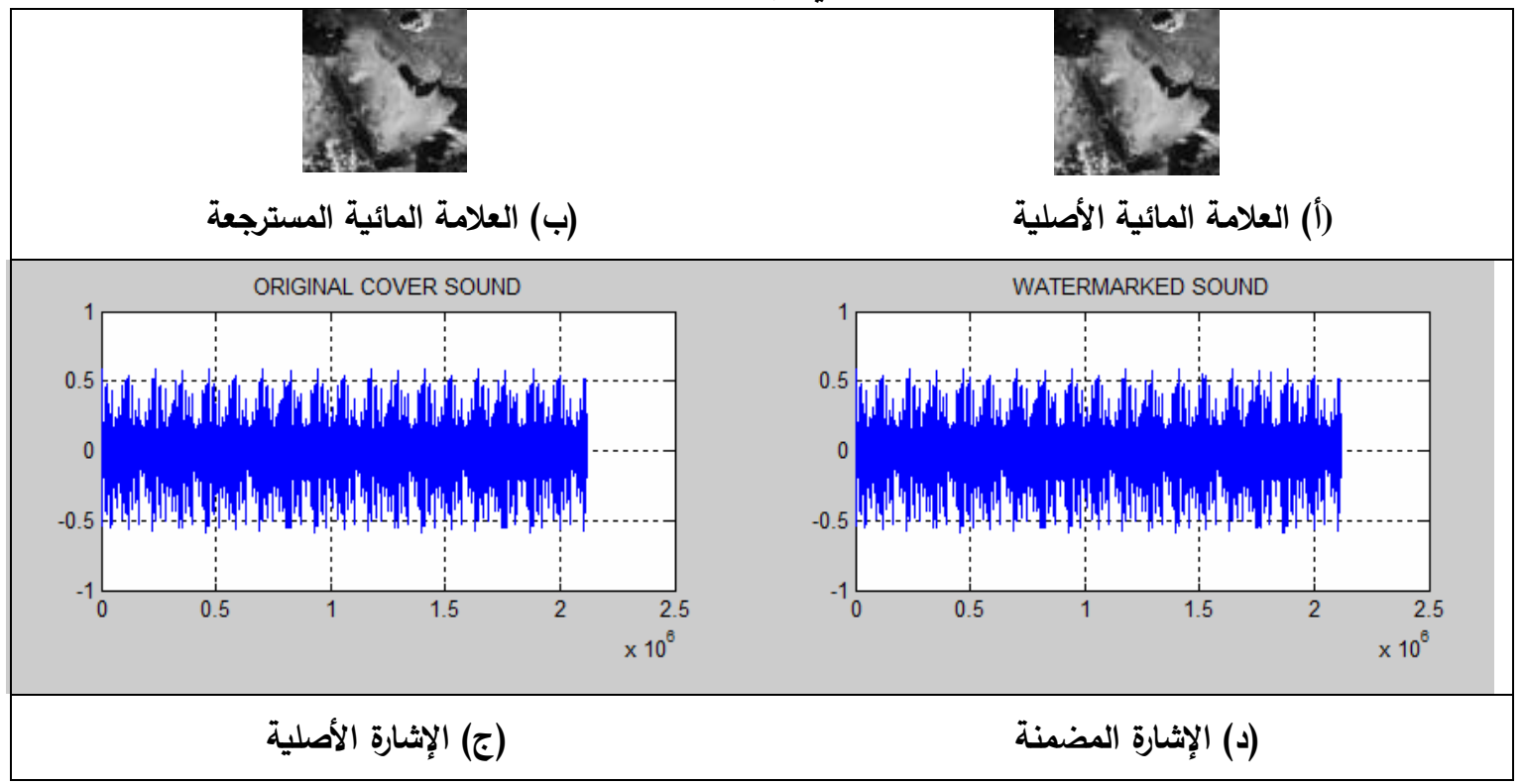

الثكل(9) تضمين صورة رمادية (L_map.bmp) في إثارة صوتية (loopmusic1.wav) 


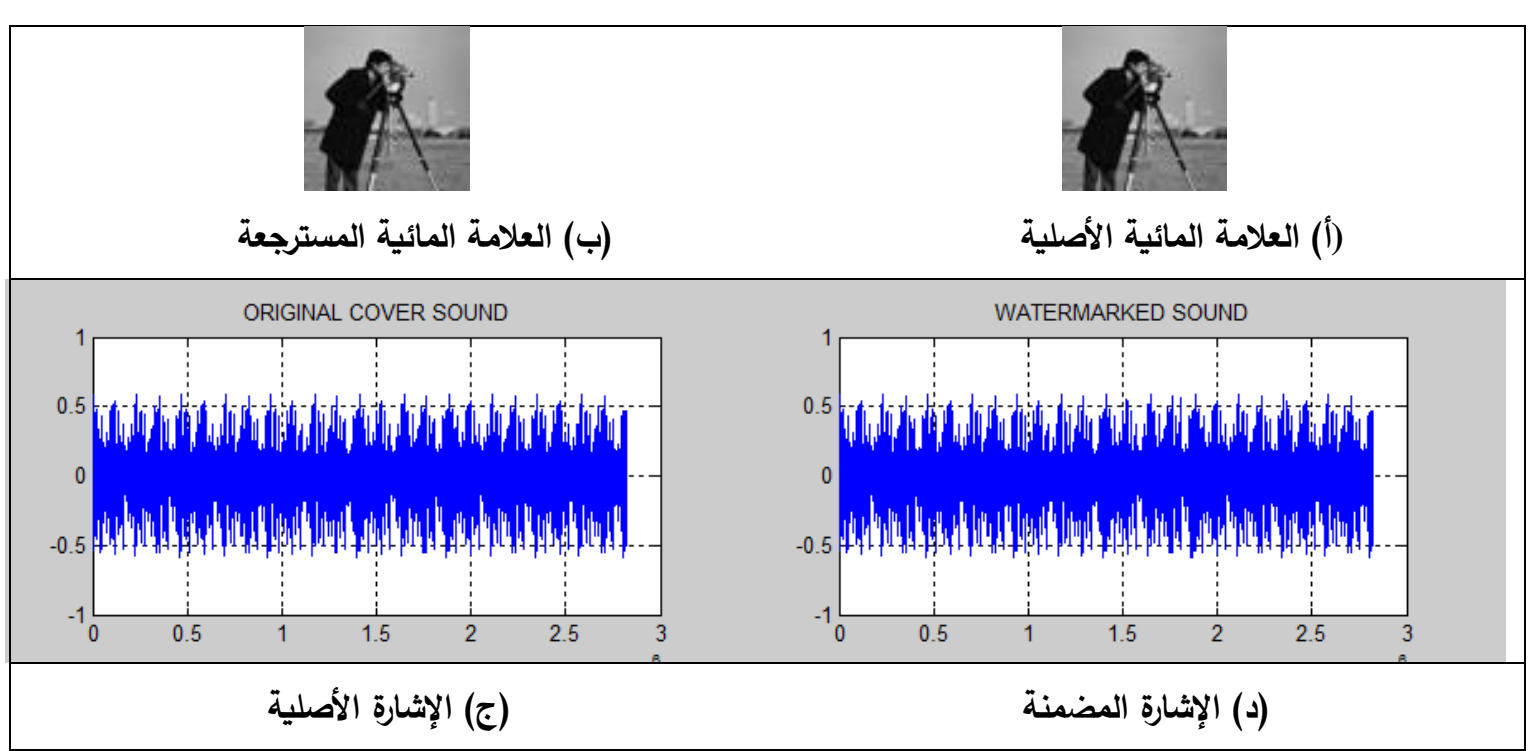

الثكل(10) تضمين صورة رمادية (cameraman.bmp) في إثارة صوتية (loopmusic1.wav)

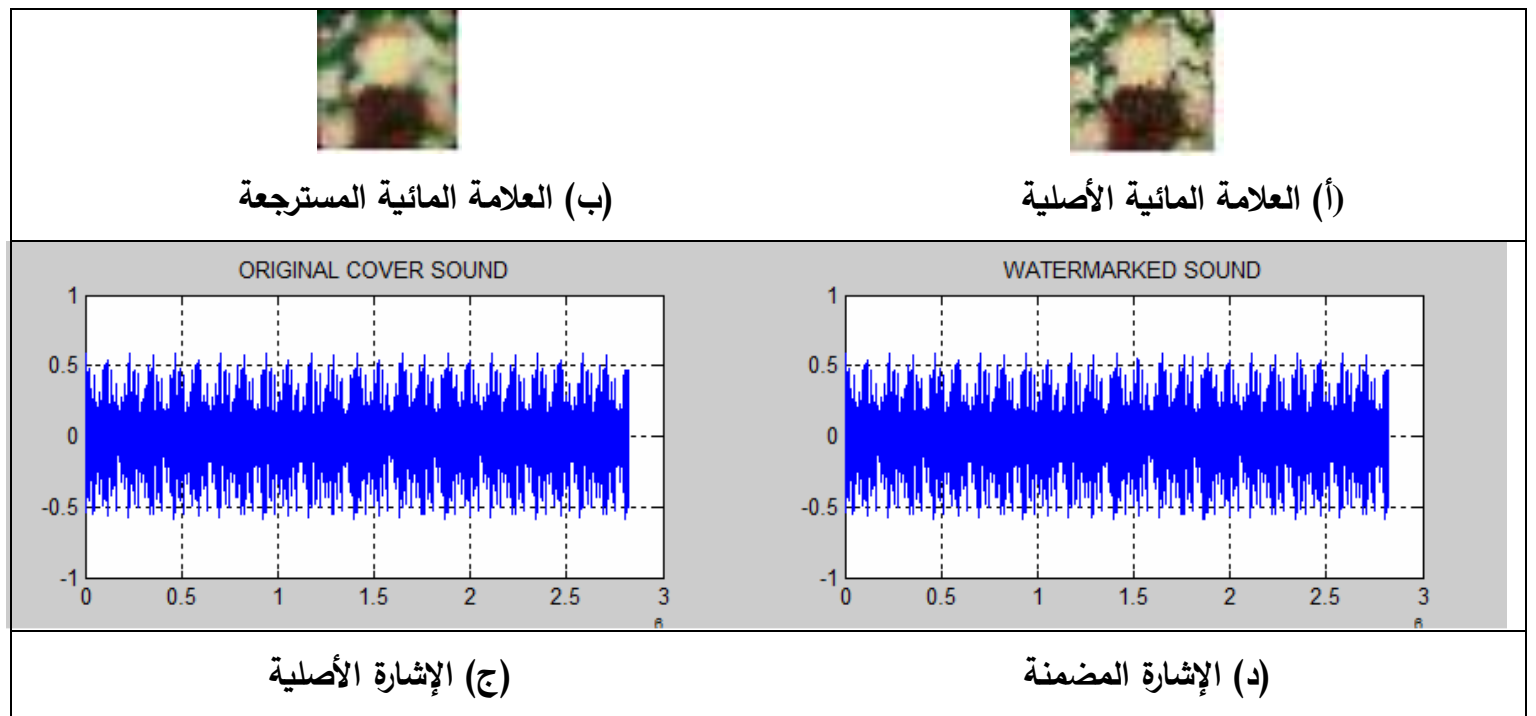

(loopmusic1.wav) في إثشارة صوتية (Swar117.jpg) الثكل (11) تضمين صورة ملونة

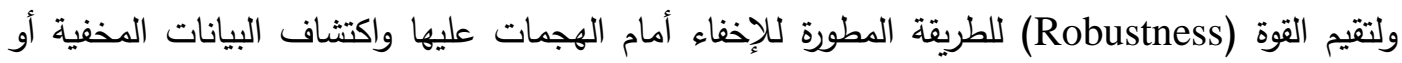
تدميرها و تثويشها تم تعريض إثارة الصوت المضمنة بداخلها العلامة المائية إلى العديد من الهجمات ومن ثم تم قياس مقدار معامل الارتباط بين الصورة الأصلية والصورة المسترجعة وفيما يلي عرض لانواع الهجمات التي تم تتفيذها على الإثارة المضمنة بداخلها العلامة المائية. 1. إضـافة الضوضـاء (Add noise) : تم إضافة سلسلة من الضوضـاء البيضـاء (white noise) إلى الإشارة $Z^{\prime}(n)=x^{\prime}(n)+A \cdot g(n)$

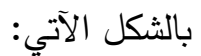

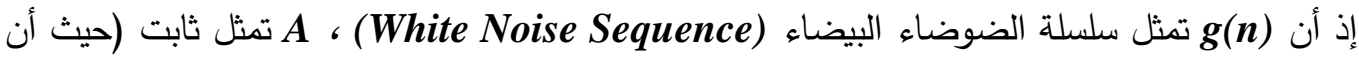

قيمة A =0.08) ، (n) تمثل الإثارة المضمنة بداخلها العلامة المائية. 2. التضخيم (Amplify) : توسيع سعة (Amplitude) الإثارة المضمن بداخلها العلامة المائية. 3. zero cross : تصفير قيم العينات الأقل من قيمة عتبة معينة. 
والجدول (3) يعرض الصورة المسترجعة بعد تعريض الإشارة إلى الهجمات المذكورة سابقا.وكذلك يبين نسبة

معامل الارتباط.

الجدول (3) نتائج الصورة المسترجعة بعد تعريض الإثارة الصوتية إلى الهجمات

\begin{tabular}{|c|c|c|}
\hline قياس جودة الصورة & & \\
\hline $\begin{array}{c}\text { CORR } \\
\text { الصورة الأصلية/لصورة المسترجعة } \\
\text { الصورة }\end{array}$ & صورة العلامة المائية المسترجعة & ن الهجوم \\
\hline 0.97551 & & Add noise \\
\hline 0.97448 & & Zero cross \\
\hline 0.91486 & & Amplitude \\
\hline
\end{tabular}

9. - 9. 9 الاستنتاجات

تم التوصل إلى الاستتناجات الآتية:-

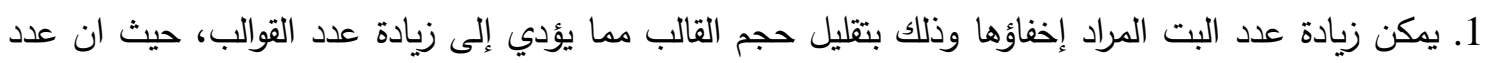
البت الممكن إخفاؤها يعتمد على عدد القوالب.

2. على الرغم من استخدام DWT (حيث يحقق التحويل المويجي كبس بفقدان ) و التي تحقق نسبة كبس للإثارة

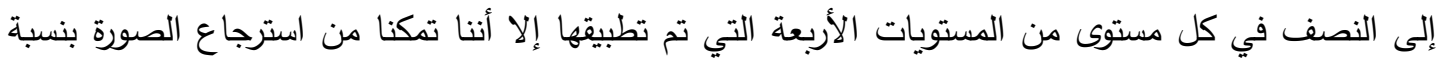
جيدة .

3. التضمين في عناصر المصفوفة V يعطي قوة أكثر (robustness) ضد الضوضاء من التضمين في العناصر القطرية للمصفوفة S المطبق في بحوث أخرى.

4. إن تأثير حدوث الخطأ (في حالة حصوله) في الصورة الملونة مدكن أن يكون كبيراً حسب موقع البت حيث أن القيمة اللونية للصورة الملونة تمثل ب(24) بت. ومن خلال التطبيق العملي لاحظنا استرجاع الصورة الملونة بصورة جيدة حيث إن معامل الارتباط المحسوب يمثل علاقة خطية قوية جدا وهذا دليل على أن نسبة الخطأ في قيم البت المسترجع قليل جدا ويكاد يكون معدوماً. 5. على الرغم من الإخفاء في الخلية الثنائية الأعلى أهمية MSB داخل العنصر للمصفوفة V فان قيمة SNR فئست بين الإثارة الأصلية والإثارة بعد التضمين و حسب الجدول رقم(2) المبين سابقا كانت جيدة. 
6. نتائج الخوارزمية بعد تطبيق عدد من الهجمات على الإشارة المضمنة كانت جيدة كما مبين في الجدول رقم (3) وهذا يدل على قوة الخوارزمية المطورة.

10. الأعمال المستقبلية

1. يمكن تطبيق الخوارزمية المطورة على ملفات الفيديو(الغطاء).

2. تشفير العلامة المائية قبل إجراء عملية التضمين لزيادة السرية.

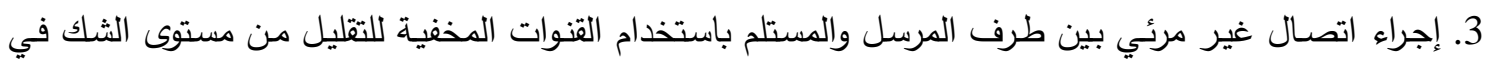

وجود اتصال سري. 


$$
\begin{aligned}
& \text { المصادر } \\
& \text { الحمامي، عـلاء حسين و الحمامي، محمد علاء (2008)، "إخفاء المعلومات: الكتابة المخفية والعلامـة } \\
& \text { المائية "، إثراء للنشر والتوزيع، الثارقة. } \\
& \text { العلي، فرح طارق محمد (2004)، "حماية استتساخ الكتابة باستخدام انتشار الطيف"، رسالة ماجستير } \\
& \text { غير منشورة، قسم علوم الحاسبات، كلية علوم الحاسبات والرياضيات، جامعة الموصل، العراق. }
\end{aligned}
$$

[3] Al_Haj Ali, and Mohammad Ahmad, (2010), "Digital Audio Watermarking Based on the Discrete Wavelets Transform and Singular Value Decomposition", European Journal of Scientific Research, ISSU 1450-216X Vol. 39 No1,pp. 621.

[4] Ali, Dujan Basheer Taha, (2004), " Digital Image Watermarking Techniques for Copyright Protection ", Unpublished D. Ph. Thesis, University of Mosul, Iraq.

[5] Amansouri, A mahmoudi Aznaveh and Ftorkamani , (2009), " SVD_Based Digital Image Watermarking Using Complex Wavelet Transform ", Sadhaua Vol. 34,part3,PP. 393_406@ printed in India.

[6] Chandra DVS,( 2002)," Digital image watermarking using singular value decomposition ",IEEE Midwest Symposium on Circuits and Systems Tulsa Oklahoma,III 264-III 267.

[7] Donaled G.Childers, (2000), " Speech Processing and Synthesis Tool Boxes ", United States of America.

[8] Kashiappa Aditya,( 2010), " Acclerating Watermarking in SVD Domain Using Graphic Processing Units ", M.Sc Thesis, Unversity Massachusetts Dartmouth.

[9] Rocha A., and Goldenstein S., (2008), "Steganography and Steganalysis in Digital Multimedia: Hype or Hallelujah?", RITA,Vol. 15, No. 1,pp. 83-110.

[10] Saffor, Amhamed and Ramli, Abdul Rahman, (2001), "A Comparative Study of Image Compression between JPEG and Wavelet", University Putra Malaysia, vol. 14, no. 1, 2001, pp. 39-45, Selangor, Malaysia.

[11] Shen. D, Lu. Z \& Pharmaceuticals. A, "Computation of Correlation Coefficient and Its Confidence Interval in SAS", Paper 170-31, www.sas.comproceedingssugi31170-31.pdf.

[12] Wu,Y, (2009), "on the Security of SVD_Based Ownership Watermarking", Multimedia 7,PP. 624_627. 